\title{
Análise das atuais evidências sobre a dor pós-operatória ao tratamento endodôntico: revisão de literatura
}

Analysis of current evidence on post-operative pain for endodontic treatment: literature review

Análisis de la evidencia actual sobre el dolor posoperatorio al tratamiento endodóntico: revisión de la literatura

Recebido: 07/09/2021 | Revisado: 17/09/2021 | Aceito: 27/09/2021 | Publicado: 29/09/2021

Bárbara Thayllane da Silva Santos ORCID: https://orcid.org/0000-0002-5104-9854 Faculdade Herrero, Brasil E-mail: barbara.thayllane@hotmail.com

Daniel Pinto de Oliveira

ORCID: https://orcid.org/0000-0003-1992-9920 Universidade Federal de Alagoas, Brasil E-mail: dpoendo@yahoo.com.br

Clóvis Stephano Pereira Bueno ORCID: https://orcid.org/0000-0001-9149-1674 Faculdade Herrero, Brasil E-mail: clovisspbueno@gmail.com

Leopoldo Cosme Silva

ORCID: https://orcid.org/0000-0002-5755-1933 Universidade Federal de Alagoas, Brasil

E-mail: leopoldocosme@gmail.com

Phillip Lucas Ricardo Pereira

ORCID: https://orcid.org/0000-0003-0911-4672 Faculdade Herrero, Brasil

E-mail: phillippereiraa@gmail.com

Laís Christina Pontes Espíndola

ORCID: https://orcid.org/0000-0002-1023-7891

Universidade Federal de Alagoas, Brasil

E-mail: laisespindola@ hotmail.com

Dyana dos Santos Fagundes

ORCID: https://orcid.org/0000-0002-7427-4114

Universidade Federal de Alagoas, Brasil

E-mail: dydyana_fagundes@hotmail.com

\begin{abstract}
Resumo
A dor pós-operatória ao tratamento endodôntico é de origem multifatorial e pode afetar a qualidade de vida do paciente. Uma variedade de técnicas e combinações podem alcançar o controle da dor durante os procedimentos endodônticos. Ter conhecimento desses fatores guia o dentista em sua capacidade de aumentar a confiança do paciente, conduzir o tratamento de acordo com os fatores de risco para a dor e tomar medidas para reduzir os sintomas. Dessa forma, o objetivo deste trabalho foi analisar as evidências atualmente disponíveis sobre o tema dor pós-operatória ao tratamento endodôntico. Para sua concretização foi realizado um levantamento na plataforma PubMed e incluídos artigos de revisão sistemática publicados entre 2016 e 2021. Os resultados deste estudo mostraram que a administração da medicação pré-operatória, a manutenção da patência apical, a agitação da substância irrigante com dispositivos automatizados e o uso de crioterapia intracanal são favoráveis na prevenção e/ou redução dos sintomas pós-operatórios. No entanto, faz-se necessário novos ensaios clínicos randomizados bem desenhados para melhor esclarecimento de alguns fatores relacionados à algesia após o tratamento endodôntico.
\end{abstract}

Palavras-chave: Dor pós-operatória; Tratamento do canal radicular; Endodontia.

\begin{abstract}
Postoperative pain after endodontic treatment has a multifactorial origin and can affect the patient's quality of life. A variety of techniques and combinations can achieve pain control during endodontic procedures. Being aware of these factors guides the dentist in his ability to increase patient confidence, conduct treatment according to risk factors for pain, and take steps to reduce symptoms. Thus, the objective of this study was to analyze the evidence currently available on the topic of postoperative pain to endodontic treatment. For its realization, a survey was carried out on the PubMed platform and systematic review articles published between 2016 and 2021 were included. The results of this study showed that the administration of preoperative medication, maintenance of apical patency, agitation of the
\end{abstract}


irrigating substance with automated devices and the use of intracanal cryotherapy are favorable in preventing and/or reducing postoperative symptoms. However, new well-designed randomized clinical trials are needed to better clarify some factors related to algesia after endodontic treatment.

Keywords: Postoperative pain; Root endodontic therapy; Endodontics.

\section{Resumen}

El dolor postoperatorio tras el tratamiento endodóntico tiene un origen multifactorial y puede afectar la calidad de vida del paciente. Una variedad de técnicas y combinaciones pueden lograr el control del dolor durante los procedimientos de endodoncia. Ser consciente de estos factores guía al dentista en su capacidad para aumentar la confianza del paciente, realizar el tratamiento de acuerdo con los factores de riesgo del dolor y tomar medidas para reducir los síntomas. Así, el objetivo de este estudio fue analizar la evidencia actualmente disponible sobre el tema del dolor postoperatorio al tratamiento endodóntico. Para su realización se realizó una encuesta en la plataforma PubMed y se incluyeron artículos de revisión sistemática publicados entre 2016 y 2021. Los resultados de este estudio mostraron que la administración de medicación preoperatoria, mantenimiento de la permeabilidad apical, agitación de la sustancia irrigante con automatismos Los dispositivos y el uso de crioterapia intracanal son favorables para prevenir y/o reducir los síntomas posoperatorios. Sin embargo, se necesitan nuevos ensayos clínicos aleatorios bien diseñados para aclarar mejor algunos factores relacionados con la algesia después del tratamiento endodóntico.

Palabras clave: Dolor postoperatorio; Tratamiento del conducto radicular; Endodoncia.

\section{Introdução}

O tratamento dos canais radiculares é um procedimento comum na prática odontológica. As principais causas pelas quais a terapia endodôntica é indicada são a inflamação persistente da polpa dentária e a presença de necrose pulpar causada por cáries, traumas dentários ou trincas (Manfredi et al., 2016). O tratamento visa reverter a doença e, assim, eliminar os sintomas associados. No entanto, quando o próprio procedimento endodôntico inicia o aparecimento da dor, o resultado acaba sendo desconfortante para o paciente e para o operador (Sathorn et al., 2008).

A dor pós-operatória é de origem multifatorial e pode afetar a qualidade de vida do paciente (Nagendrababu et al., 2017; Arias et al., 2013). A incidência da dor após o tratamento endodôntico varia de 3\% a 58\% (Sathorn et al., 2008) e, embora a algesia diminua após a intervenção, sintomas residuais podem permanecer devido aos efeitos da inflamação (Shirvani et al., 2017).

Uma variedade de técnicas e combinações podem alcançar o controle da dor durante os procedimentos endodônticos (Suneelkumar et al., 2018). Ter conhecimento sobre esses fatores guiará o dentista em sua capacidade de aumentar a confiança do paciente, conduzir o tratamento de acordo com os fatores de risco para a dor e tomar medidas para reduzir os sintomas (Nagendrababu et al., 2017). No entanto, prevenir o desenvolvimento e obter o alívio da dor pós-endodôntica continua sendo um desafio para o endodontista (Suneelkumar et al., 2018). Uma das principais preocupações em relação ao estudo da dor é a sua forma de avaliação, visto que a dor é uma experiência sensorial, subjetiva e intrapessoal cujo limiar varia entre os sujeitos (Pasqualini et al., 2012).

A correlação dos sintomas pós-operatórios com diferentes variáveis, como o número de visitas necessárias para completar o tratamento de canal, o uso de pré-medicação oral, o tipo de instrumento e os procedimentos intra-operatórios realizados têm sido o foco de vários estudos (Manfredi et al., 2016; Nagendrababu et al. 2017; Suneelkumar et al., 2018; Schwendicke et al. 2017; De Geus et al., 2018; Nagendrababu et al., 2019; Sun et al., 2018; Martins et al., 2019). Muitos são os fatores que explicam a dor pós-operatória e estes são bastante variáveis entre os trabalhos. Assim, o principal objetivo desta revisão de literatura foi analisar as evidências atualmente disponíveis acerca do tema dor pós-operatória após o tratamento do canal radicular, através de revisões sistemáticas realizadas nos últimos cinco anos.

\section{Metodologia}

Com base na metodologia de Koche (2011), para a execução desta revisão de literatura foi realizada uma busca na 
plataforma online PubMed, utilizando os descritores "postoperative pain" AND "root endodontic therapy" AND “endodontics”, identificando inicialmente 474 estudos. Ao aplicar o filtro 'Revisão Sistemática', os resultados diminuíram para 38 artigos. Por fim, ao limitar a data de publicação para os últimos cinco anos, obteve-se um total de 27 trabalhos. Como critério de inclusão, foram incluídos os artigos que, após a leitura do resumo, abordaram o tema dor pós operatória ao tratamento endodôntico primário e tratavam de revisão sistemática. Como critério de exclusão, foram excluídos os artigos que não tratavam do tema, que não eram revisão sistemática, que não estudavam os dentes permanentes e os que abordaram retratamento e/ou tratamentos endodônticos cirúrgicos. Após a seleção dos artigos de acordo com os critérios, 8 trabalhos foram excluídos; portanto, 19 estudos foram incluídos nesta revisão bibliográfica (figura 1).

Figura 1: Metodologia utilizada para obtenção dos estudos.

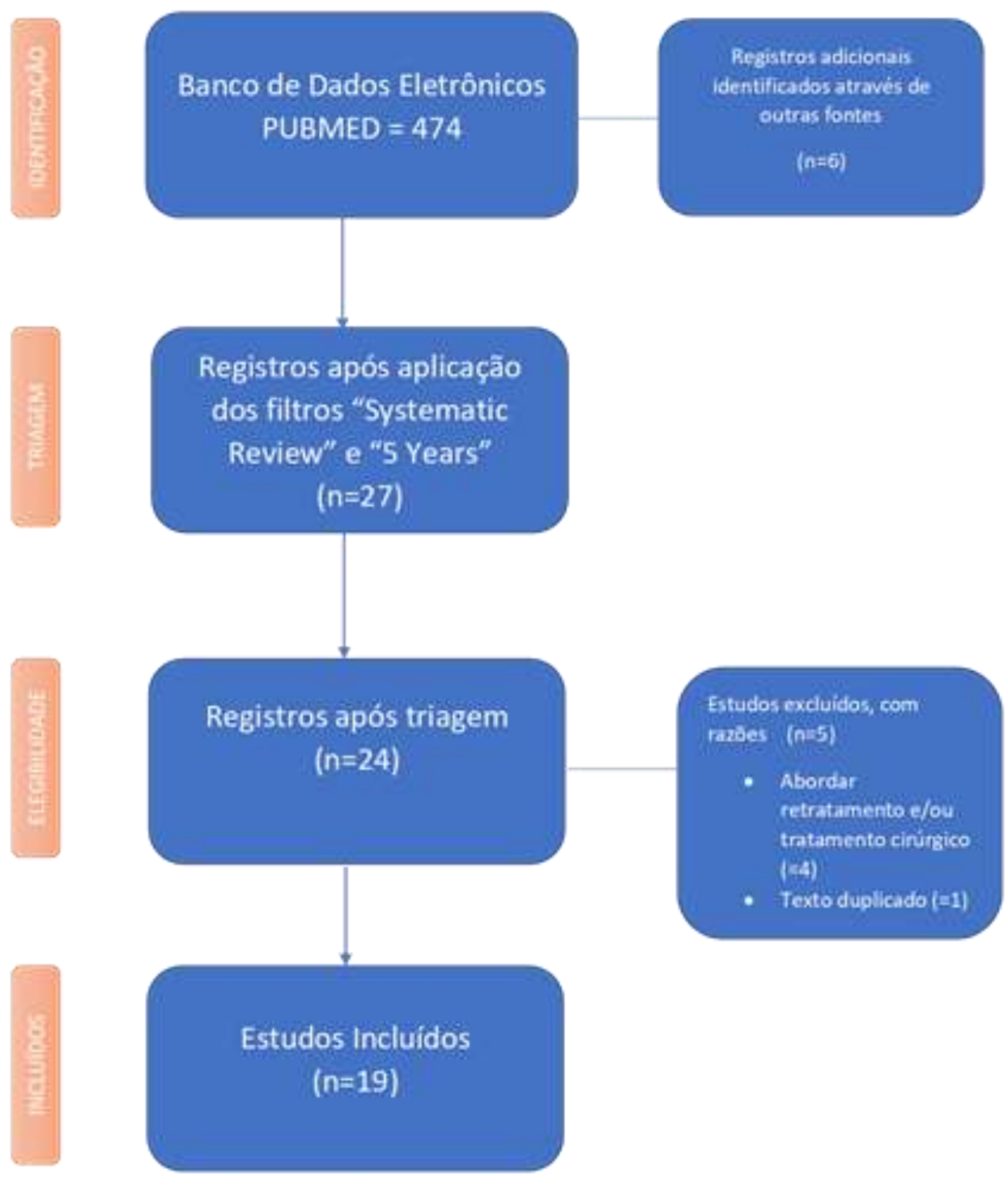

Fonte: Pesquisa direta (2021).

\section{Resultados e Discussão}

\subsection{Visita única $X$ múltiplas visitas}

Visto que uma das causas da dor pós-operatória pode estar relacionada com o número de visitas na realização do tratamento endodôntico, Manfredi et al. (2016) realizaram um estudo de revisão sistemática com o objetivo de determinar se o 
número de sessões para a conclusão do tratamento de canal faz diferença em termos de eficácia e complicações. Ao avaliar a intensidade dos sintomas em diferentes intervalos de tempo, os autores concluíram que a incidência de dor após o tratamento endodôntico é semelhante, independente do número de visitas; embora, pacientes submetidos ao procedimento de sessão única possam sentir um nível mais elevado de dor no período de uma semana pós-tratamento e sejam mais propensos a tomar analgésicos.

De acordo com o estudo de Schwendicke et al. (2017), o risco de complicações a curto e longo prazo após o tratamento endodôntico não foi significativamente diferente entre os procedimentos de sessão única e múltipla. No entanto, quando analisado o risco de flare up entre os dois grupos, o risco da complicação acontecer foi maior após procedimentos de visita única; por isso, os autores recomendam o tratamento em múltiplas sessões de dentes que apresentam risco aumentado, como dentes com lesões periapicais existentes.

Nagendrababu et al. (2017) avaliaram os fatores que influenciam nos sintomas pós-operatórios após o tratamento de canal radicular em visita única. Conforme os achados, uma maior incidência de dor pós-operatória foi observada quando os sintomas pré-operatórios estavam presentes e o tratamento de canal foi realizado em única sessão; também, foi observado que nos casos em que a dor pré-operatória existia, os sintomas no pós-tratamento foram maiores quando a patência apical foi mantida. Ao avaliar os aspectos radiográficos, um estudo informou que a presença de uma radioluscência periapical é capaz de reduzir a dor pós-operatória.

Com base nas evidências, o número de sessões não parece ter relação na intensidade da dor após a terapia endodôntica. No entanto, na abordagem de múltiplas visitas, a incidência de edema foi semelhante entre os dois grupos quando nenhuma medicação intracanal foi empregada entre as consultas, enquanto, quando o hidróxido de cálcio foi deixado nos canais entre as sessões, o tratamento de múltiplas consultas teve um desempenho superior (Manfredi et al., 2016).

No estudo de Schwendicke et al. (2017) foi observado que tratamentos realizados em visita única têm um risco maior de ocorrer flare up, principalmente em dentes com lesões apicais pré-existentes. Em contrapartida, Nagendrababu et al. (2017) observaram uma menor prevalência de dor em pacientes que apresentaram lesões periapicais radiográficas; segundo Ng et al. (2004), isso pode ser atribuído ao fato de que dentes com lesões periapicais grandes ( $>3 \mathrm{~mm}$ ) atuam como um tampão contra o aumento da pressão do exsudato durante a resposta inflamatória a lesões mecânicas ou químicas; porém, esse parâmetro de avaliação foi feito por radiografias convencionais, ao passo que futuros estudos com tomografias computadorizadas podem resultar em observações e desfechos diferentes (Nagendrababu et al., 2017).

\subsection{Uso de medicação}

Para avaliar o efeito da medicação pré-operatória na dor pós-endodôntica, Suneelkumar et al. (2018) examinaram o uso de corticosteróides em pacientes com pulpite irreversível sintomática submetidos ao tratamento endodôntico de sessão única. Os resultados mostraram que houve uma maior redução da dor pós-operatória em curto prazo quando doses profiláticas de corticosteróides foram administradas. A administração pré-operatória de prednisolona $30 \mathrm{mg}$ e dexametasona antes do procedimento de sessão única resultou em menor incidência de dor.

Conforme o estudo de Nagendrababu et al. (2019), os corticosteróides foram classificados como o melhor tratamento para a redução da dor nos intervalos de 6, 12 e 24 horas após o procedimento. Segundo os autores, a pré-medicação oral é capaz de reduzir os sintomas pós-operatórios ao tratamento de canal radicular não cirúrgico, sendo o piroxicam ou prednisolona a pré-medicação de escolha.

Já De Geus et al. (2018) avaliaram o efeito do ibuprofeno como pré-medicação oral na redução da dor pós-operatória. Os resultados apresentaram nenhuma diferença significativa na intensidade da dor ao compará-lo com placebo ou outras drogas; portanto, segundo os autores, não há evidências claras de que o ibuprofeno pré-operatório seja melhor que outros 
medicamentos na redução do risco e intensidade da dor pós-endodôntica.

Contrariando os resultados do estudo anterior, Smith et al. (2017) relataram que o ibuprofeno na posologia de $600 \mathrm{mg}$ se mostrou eficaz no alívio da dor no período de 6 horas após o tratamento endodôntico, quando comparado ao placebo. Além disso, a combinação de ibuprofeno $600 \mathrm{mg}$ + paracetamol $1000 \mathrm{mg}$ pareceu ser significativamente mais eficaz. O ibuprofeno e o paracetamol são sinérgicos (Miranda et al., 2006), o que pode ter favorecido seus resultados quando associados.

Contudo, no trabalho de Smith et al. (2017) houve uma ausência de padronização dos estudos quanto ao momento da administração do medicamento, realizadas no período pré e pós-tratamento, enquanto, no trabalho de De Geus et al. (2018) o ibuprofeno foi administrado de maneira profilática, o que justificaria, talvez, a divergência dos resultados entre os trabalhos.

Por fim, o estudo de Shirvani et al. (2017) teve como objetivo sintetizar a eficácia de analgésicos não narcóticos no tratamento da dor endodôntica pós-operatória. Os resultados mostraram uma redução significativa nos escores de dor nos grupos tratados com os analgésicos não narcóticos. Além disso, os achados mostraram maior magnitude do efeito em pacientes com pulpite irreversível. Esse grupo farmacológico é capaz de reduzir a dor inflamatória e, por isso, os resultados foram favoráveis para pacientes com pulpite (Shirvani et al., 2017).

Segundo Shirvani et al. (2017), a administração de analgésicos não narcóticos após o tratamento endodôntico pareceu melhorar a analgesia em até 24 horas; porém, isso pode estar relacionado com o fato de a intensidade da dor diminuir com o tempo (Shirvani et al., 2017). Sabe-se que 1 hora após a terapia endodôntica ocorre um pico de dor, que está relacionado exclusivamente à presença de inflamação, porém, após o primeiro dia há uma diminuição significativa da dor (Martins et al., 2019).

\subsection{Instrumentos endodônticos}

A revisão sistemática realizada por Sun et al. (2018) teve como objetivo avaliar duas condições: equiparar a dor pósoperatória utilizando instrumentos manuais e rotatórios e comparar a dor pós-tratamento entre instrumentos rotatórios e reciprocantes. Os resultados mostraram que o uso de instrumentos rotatórios contribuiu para menor incidência e intensidade de dor pós-operatória em comparação às limas manuais. Além disso, o uso de diferentes sistemas rotatórios contribuiu para uma menor ocorrência de dor em relação aos reciprocantes.

Em contrapartida, o estudo de Martins et al. (2019) mostrou que o movimento rotatório parece impactar negativamente na dor pós-operatória em relação à cinemática reciprocante; além disso, mais pacientes apresentaram dor intensa após 48 horas com o movimento rotatório contínuo em comparação com o outro.

Um dos fatores responsáveis pela dor pós-operatória pode ser o preparo químico-mecânico dos canais radiculares (Nagendrababu et al., 2017; Sun et al., 2018), pois, todas as técnicas de instrumentação endodôntica disponíveis são capazes de extruir debris através do forame (Sathorn et al., 2008). De acordo com Sun et al. (2018), além da extrusão de debris para a região apical, outros motivos podem explicar as diferenças na dor causadas por diferentes instrumentos, como a quantidade de debris remanescentes no canal radicular, tamanho do preparo apical, tempo de operação, entre outros. No entanto, os dois trabalhos analisados neste estudo forneceram resultados contrastantes, dessa forma, outros estudos de revisão sistemática comparando as cinemáticas rotatória e reciprocante na dor pós-operatória são necessários.

\subsection{Alargamento foraminal}

A instrumentação do terço apical é considerada uma etapa importante no tratamento do canal radicular, dessa forma, as substâncias irrigantes conseguem penetrar na extremidade apical da raiz, auxiliando na limpeza e mantendo o trajeto livre de debris (Yaylali et al., 2018).

Dessa forma, Silva et al. (2017) conduziram um estudo de revisão sistemática com o objetivo de investigar se o 
alargamento foraminal causa sintomas pós-operatórios diferentes em comparação ao tratamento endodôntico convencional. Os resultados demonstraram predomínio de dor pós-operatória nos estudos que executaram a técnica com instrumentos mecanizados. O trabalho que utilizou limas reciprocantes relatou uma baixa incidência de dor, enquanto o estudo que avaliou o sistema rotatório observou uma diferença significativa na dor pós-tratamento nos primeiros dois dias. Além disso, os autores concluíram que a dor pós-operatória foi maior nos primeiros dias quando o alargamento foraminal foi realizado em dentes necrosados e com periodontite apical.

\subsection{Patência apical}

Abdulrab et al. (2018) avaliaram o efeito da patência apical durante o tratamento endodôntico, considerando a dor pós-operatória como desfecho principal. Os resultados revelaram não haver diferença significativa na dor pós-operatória entre a manutenção da patência apical e a não manutenção nos primeiros dias após o tratamento; mas, demonstrou menos dor pósoperatória no quinto dia quando a técnica foi realizada. Com isso, os autores concluíram que a manutenção da patência apical não aumenta a dor pós-operatória, mas que pode melhorá-la.

Do mesmo modo, Yaylali et al. (2018) relataram que, quando avaliado o efeito da patência apical em dentes vitais e não vitais, não houve aumento na dor pós-operatória quando comparada ao grupo controle e a taxa de flare up foi de $0 \%$. Além disso, um estudo relatou haver menos dor pós-operatória quando a patência foi mantida.

A limpeza não adequada dessa área poderá servir como causa potencial de infecção persistente, comprometendo o resultado do tratamento (Silva et al., 2017). Os dois estudos incluídos no presente trabalho concordam que a realização da patência apical não aumenta a dor pós-operatória, mas que é capaz de reduzi-la; no entanto, essa conclusão deve ser considerada com cautela devido às variações presentes nos estudos.

\subsection{Glide path}

Cunha et al. (2020) compararam a influência da cinemática de glide path na ocorrência da dor pós-operatória. Os trabalhos selecionados equipararam a técnica de execução do glide path com diferentes instrumentos (manuais, rotatórios e reciprocantes) e a não execução da técnica, para avaliar a existência da dor após o preparo do canal radicular. Os resultados deste estudo relataram níveis de dor mais baixos na cinemática de glide path com os instrumentos mecanizados (rotários e reciprocantes) em comparação com as limas manuais; além disso, foram encontrados resultados superiores para a cinemática rotatória em relação ao grupo que não realizou o glide path. Ao equiparar as cinemáticas rotatória e reciprocante na dor pósoperatória, diferenças significativas não foram encontradas.

\subsection{Agitação intracanal automatizada}

Com o objetivo de avaliar se a agitação da substância irrigante com dispositivos automatizados resulta em menos dor pós-operatória em relação à irrigação convencional com seringa e agulha, Decurcio et al. (2019) realizaram um estudo de revisão sistemática. Os resultados demonstraram que a agitação automatizada reduz a dor pós-tratamento em 24 e 48 horas, principalmente em dentes diagnosticados com pulpite irreversível sintomática e dor pré-operatória de moderada a intensa. As possíveis razões para a técnica em estudo ser favorável na redução da dor podem estar relacionadas ao movimento da solução irrigante para a direção cervical, reduzindo o risco de extrusão e danos à região apical (Decurcio et al., 2019).

\subsection{Redução oclusal}

Nguyen et al. (2019) analisaram a relação entre redução oclusal e a dor pós-operatória no tratamento dos canais radiculares. Os autores sugerem que a redução oclusal possa ser mais relevante no tratamento endodôntico de pacientes que 
apresentam parafunção oclusal e são diagnosticados com pulpite irreversível ou periodontite apical sintomática; não parecendo ser favorável na redução da sintomatologia em dentes com dor pré-operatória leve. Segundo os autores, a eficácia da técnica na redução da dor parece ser restrita ao sexto dia em dentes diagnosticados com pulpite irreversível.

De acordo com o estudo de Shamszadeh et al. (2020), a redução oclusal pode ser benéfica a partir do terceiro dia póstratamento, em dentes que apresentam dor endodôntica pós-operatória leve a moderada.

O cirurgião-dentista deve considerar o benefício e as sequelas para a execução da técnica, visto que a remoção do esmalte ou dentina da superfície dental pode ser suficiente para alterar a oclusão do paciente e alertar para a necessidade de novas restaurações (Nguyen et al., 2019).

\subsection{Crioterapia intracanal}

A crioterapia intracanal consiste em um método que utiliza a aplicação de frio durante o tratamento, sendo considerada uma alternativa eficaz para o controle da dor pós-operatória. Sendo assim, Sadaf et al. (2020) avaliaram o efeito da técnica na redução dos sintomas pós-operatórios. Os resultados obtidos mostraram que a crioterapia intracanal é capaz de reduzir significativamente a intensidade da dor em 6 e 24 horas após a aplicação da técnica. Os autores concordam que a presença de dor pré-operatória intensa é um fator modificador significativo no efeito positivo da crioterapia. Além disso, os resultados sugerem que a técnica pode ser mais eficaz em dentes diagnosticados com periodontite apical.

De acordo com o estudo anterior, Monteiro et al. (2020) concordam que o efeito da crioterapia na redução da dor pósoperatória tem magnitude no período de 6 e 24 horas após a terapia endodôntica. Além disso, os níveis de dor após o tratamento foram significativamente mais baixos em pacientes com pulpite irreversível e periodontite apical.

Segundo Sadaf et al. (2020) e Monteiro et al. (2020), a crioterapia intracanal se mostrou favorável em dentes diagnosticados com periodontite apical, isso ocorre devido à redução da temperatura na superfície radicular externa, considerada suficiente para desacelerar a reação inflamatória, diminuir a liberação de substâncias dolorosas e diminuir o edema no tecido periapical (Alrahabi, 2017). Apesar de relatada a eficácia da técnica, os trabalhos apontam a necessidade de pesquisas adicionais, devendo-se investigar a dose e a duração ideais; assim como os efeitos adversos, que não foram relatados em qualquer estudo.

\section{Considerações Finais}

A percepção da dor é algo subjetivo e individual, cujo limiar varia entre os pacientes; portanto, mensurar sintomas pós-operatórios em estudos é passível de falhas. Com base nas evidências encontradas na maioria dos trabalhos, observou-se um número limitado de artigos para aplicação da meta-análise, além de grande parte apresentar heterogeneidade entre si. De acordo com os resultados deste estudo, a administração de medicamentos pré e pós-operatórios, a manutenção da patência apical, a agitação da substância irrigante com dispositivos automatizados e o uso de crioterapia intracanal pareceram ser favoráveis na prevenção e/ou redução dos sintomas pós-operatórios; enquanto, o alargamento foraminal parece favorecer a dor pós-operatória.

No entanto, pesquisas adicionais são necessárias, devendo-se realizar novos ensaios clínicos randomizados bem desenhados, a fim de se obter respostas acerca da dose ideal e os efeitos adversos da crioterapia intracanal; bem como, comparando as cinemáticas rotatória e reciprocante no efeito da dor pós-operatória.

\section{Referências}

Abdulrab, S., Rodrigues, J. C., Al-Maweri, S. A., Halboub, E., Alqutaibi, A. Y., \& Alhadainy, H. (2018). Effect of Apical Patency on Postoperative Pain: A Meta-analysis. J Endod, 44(10):1467-1473. 
AlRahabi, M. K. (2017). Predictors, prevention, and management of postoperative pain associated with nonsurgical root canal treatment: a systematic review. J Taibah Univ Med Sci., 12:376-84.

Arias, A., de la Macorra, J. C., Hidalgo, J. J., \& Azabal, M. (2013). Predictive models of pain following root canal treatment: a prospective clinical study. Int Endod J, 46: 784-793.

Cunha, T. C., Matos, F. S., Paranhos, L. R., Bernardino, I. M., \& Moura, C. C. G. (2020). Influence of glide path kinematics during endodontic treatment on the occurrence and intensity of intraoperative and postoperative pain: a systematic review of randomized clinical trials. BMC Oral Health, $20(1): 175$.

Decurcio, D. A., Rossi-Fedele, G., Estrela, C., Pulikkotil, S. J., \& Nagendrababu, V. (2019). Machine-assisted Agitation Reduces Postoperative Pain during Root Canal Treatment: A Systematic Review and Meta-analysis from Randomized Clinical Trials. J Endod., 45:387-393 e2.

De Geus, J. L., Wambier, L. M., Boing T. F., Loguercio, A. D., \& Reis, A. (2018). Effects of Ibuprofen Compared to Other Premedication Drugs on the Risk and Intensity of Postendodontic Pain: A Systematic Review. Eur Endod J., 3: 123-33.

Koche, J. C. (2011). Fundamentos de metodologia científica. Petrópolis: Vozes.

Manfredi, M., Figini, L., Gagliani, M., \& Lodi, G. (2016). Single versus multiple visits for endodontic treatment of permanent teeth. Cochrane Database Syst Rev., 12:CD005296.

Martins, C. M., Batista, V. E., Souza, A. C., Andrada, A. C., Mori, G. G., \& Filho, J. E. (2019). Reciprocating kinematics leads to lower incidences of postoperative pain than rotary kinematics after endodontic treatment: A systematic review and meta-analysis of randomized controlled trial. $J$ Conservative Dentistry., 22:320-31.

Miranda, H. F., Puig, M. M., Prieto, J. C., \& Pinardi, G. (2006). Synergism between paracetamol and nonsteroidal anti-inflammatory drugs in experimental acute pain. Pain, 121(1-2): 22-8.

Monteiro, L. P. B., Guerreiro, M. Y. R., Valino, R. C., Magno, M. B., Maia, L. C., \& Brandão, J. M. S. (2021). Effect of intracanal cryotherapy application on postoperative endodontic pain: a systematic review and metaanalysis. Clin Oral Investig., 25(1):23-35.

Nagendrababu, V., \& Gutmann, J. L. (2017). Factors associated with postobturation pain following single-visit nonsurgical root canal treatment: a systematic review. Quintessence Int., 48(3):193-208.

Nagendrababu, V., Pulikkotil, S. J., Jinatongthai, P., Veettil, S. K., Teerawattanapong, N., \& Gutmann, J. L. (2019). Efficacy and Safety of Oral Premedication on Pain after Nonsurgical. Root Canal Treatment: A Systematic Review and Network Meta-analysis of Randomized Controlled Trials. J Endod., 45(4):364371.

Ng, Y. L., Glennon, J. P., Setchell, D. J., et al. (2004). Prevalence of and factors affecting postobturation pain in patients undergoing root canal treatment. Int Endod J, 37: 381-391.

Nguyen, D., Nagendrababu, V., Pulikkotil, S. J., \& Rossi-Fedele, G. (2020). Effect of occlusal reduction on postendodontic pain: A systematic review and meta-analysis of randomised clinical trials. Aust Endod J., 46(2):282-294.

Pasqualini, D., Mollo L., Scotti, N., Cantatore, G., Castellucci, A., Migliaretti G., et al. (2012). Postoperative pain after manual and mechanical glide path: a randomized clinical trial. J Endod., 38:32-6.

Sadaf, D., Ahmad, M. Z., \& Onakpoya, I. J. (2020). Effectiveness of Intracanal Cryotherapy in Root Canal Therapy: A Systematic Review and Meta-analysis of Randomized Clinical Trials. J Endod., 46(12):1811-1823.

Sathorn, C., Parashos, P., \& Messer, H. (2008). The prevalence of postoperative pain and flare-up in single- and multiple-visit endodontic treatment: a systematic review. Int Endod J, 41:91-9.

Schwendicke, F., \& Göstemeyer, G. (2017). Single-visit or multiple-visit root canal treatment: systematic review, meta-analysis and trial sequential analysis. BMJ Open., 7:e013115.

Shamszadeh, S., Shirvani, A., \& Asgary, S. (2020). Does occlusal reduction reduce post-endodontic pain? A systematic review and meta-analysis. Journal of oral rehabilitation, 47(4):528-535.

Shirvani, A., Shamszadeh, S., Eghbal, M. J., \& Asgary, S. (2017). The efficacy of non-narcotic analgesics on post-operative endodontic pain: a systematic review and meta-analysis - the efficacy of non-steroidal anti-inflammatory drugs and/or paracetamol on post-operative endodontic pain. $J$ Oral Rehabil., 44(9):709-721.

Silva, E. A. B., Guimarães, L. S., Küchler, E. C., Antunes, L. A. A., \& Antunes, L.S. (2017). Evaluation of Effect of Foraminal Enlargement of Necrotic Teeth on Postoperative Symptoms: A Systematic Review and Meta-analysis. J Endod., 43(12):1969-1977.

Smith, E. A., Marshall, J. G., Selph, S. S., Barker, D. R., \& Sedgley, C. M. (2017). Nonsteroidal Anti-inflammatory Drugs for Managing Postoperative Endodontic Pain in Patients Who Present with Preoperative Pain: A Systematic Review and Meta-analysis. J Endod., 43(1):7-15.

Sun, C., Sun, J., Tan, M., Hu, B., Gao, X., \& Song, J. (2018). Pain after root canal treatment with different instruments: A systematic review and metaanalysis. Oral Dis., 24:908-19.

Suneelkumar, C., Subha, A., \& Gogala, D. (2018). Effect of preoperative corticosteroids in patients with symptomatic pulpitis on postoperative pain after single-visit root canal treatment: a systematic review and meta-analysis. J Endod., 44(9):1347-54.

Yaylali, I. E., Demirci, G. K., Kurnaz, S., Celik, G., Kaya, B. U., \& Tunca, Y. M. (2018). Does maintaining apical patency during instrumentation increase postoperative pain or flare-up rate after nonsurgical root canal treatment? A systematic review of randomized controlled trials. $J$ Endod., 44:1228-1236.) 